\title{
USO DE HERRAMIENTAS COMPUTACIONALES EN LA ENSEÑANZA Y EL APRENDIZAJE DE LA MODELACIÓN DE PROCESOS AMBIENTALES
}

APPLICATION OF COMPUTATIONAL TOOLS IN TEACHING AND LEARNING OF ENVIRONMENTAL PROCESSES MODELING

JAIME DÍAZ GÓMEZ

Ingeniero Sanitario

MSc. Environmental Science and Technology

Grupo de Investigación Gestión del Recurso Hídrico

Universidad de Boyacá, Colombia

jaimediaz@uniboyaca.edu.co

Recibido: $18 / 12 / 2012$

Aceptado: 16/05/2013 


\title{
RESUMEN
}

La modelación y simulación de un proceso biológico de lodos activados se emplea como ejemplo para ilustrar los beneficios de la aplicación de un programa computacional en la enseñanza y el aprendizaje de la Ingeniería Ambiental. La simulación se utiliza para identificar las diferencias existentes entre el proceso de lodos activados cuando es convencional y cuando usa la aireación extendida. Por medio de la simulación en el computador se identifican esas diferencias en términos de la eficiencia para remoción de materia orgánica y la configuración de los procesos variando la edad del lodo. También se pueden obtener ideas generales acerca de los costos operativos de los dos procesos. Una herramienta computacional amigable, en conjunción con la solución manual de las ecuaciones, promueve en el estudiante el planteamiento de escenarios destinados a evaluar la respuesta de los procesos ante una variación de los parámetros. Esta característica se fomenta con el uso de tal herramienta, la cual permite una simulación rápida y eficiente. Así, la modelación y la simulación con el uso de herramientas computacionales, deben ser parte fundamental de los planes de estudio de los estudiantes de ingeniería ambiental, dada su gran cantidad de beneficios.

Palabras clave: Ingeniería ambiental, procesos ambientales, currículo, simulación, modelación

\begin{abstract}
Mathematical modeling and simulation of the activated sludge biological process are used as an example to show the advantages of the application of user friendly software in the teaching and learning of environmental processes in Environmental Engineering. The simulation is used to identify the main differences between the conventional activated sludge process and the extended aeration process. Simulations are carried out using the sludge age as the operational parameter. By means of the computer simulation, it is shown that the influence of changing the sludge age allows the identification of the main differences between the processes in terms of efficiency for the removal of organic matter and its configuration. Also, general ideas about the operative cost of the two systems can be reached. The use of user-friendly software is an important tool that encourages the students to compare the manual results with the output of the computer simulation. This serves to verify theoretical concepts and draw scenarios, as well as to identify the consequences of changing the parameters of a model in a fast and efficient way. Due to the many benefits and advantages of mathematical modeling and simulation, it is evident that its use in the Environmental Engineering curriculum, by means of user-friendly software, is increasingly important
\end{abstract}

Keywords: Environmental Engineering, environmental process, curriculum, computer simulation, modeling. 


\section{INTRODUCCIÓN}

El programa de Ingeniería Ambiental de la Universidad de Boyacá ofrece una aproximación multidisciplinaria en su enseñanza. Uno de los cursos en los que el estudiante debe hacer uso de los conceptos aprendidos previamente, es el de Procesos Ambientales, el cual se enfoca en la comprensión teórica de los procesos ocurridos en sistemas ambientales. Estos sistemas pueden ser naturales (ríos, porciones de suelo, sedimentos) o de ingeniería, es decir, aquellos diseñados por el hombre para reducir el impacto de su actividad sobre el medio ambiente (Weber, 2000). En el curso, las ciencias básicas (Matemáticas, Biología, Física, Química) constituyen las bases teóricas y se emplean para construir modelos matemáticos que pueden ser implementados en programas computacionales. A su vez, la termodinámica química y la cinética química, sobresalen como pilares fundamentales (ver Figura 1).

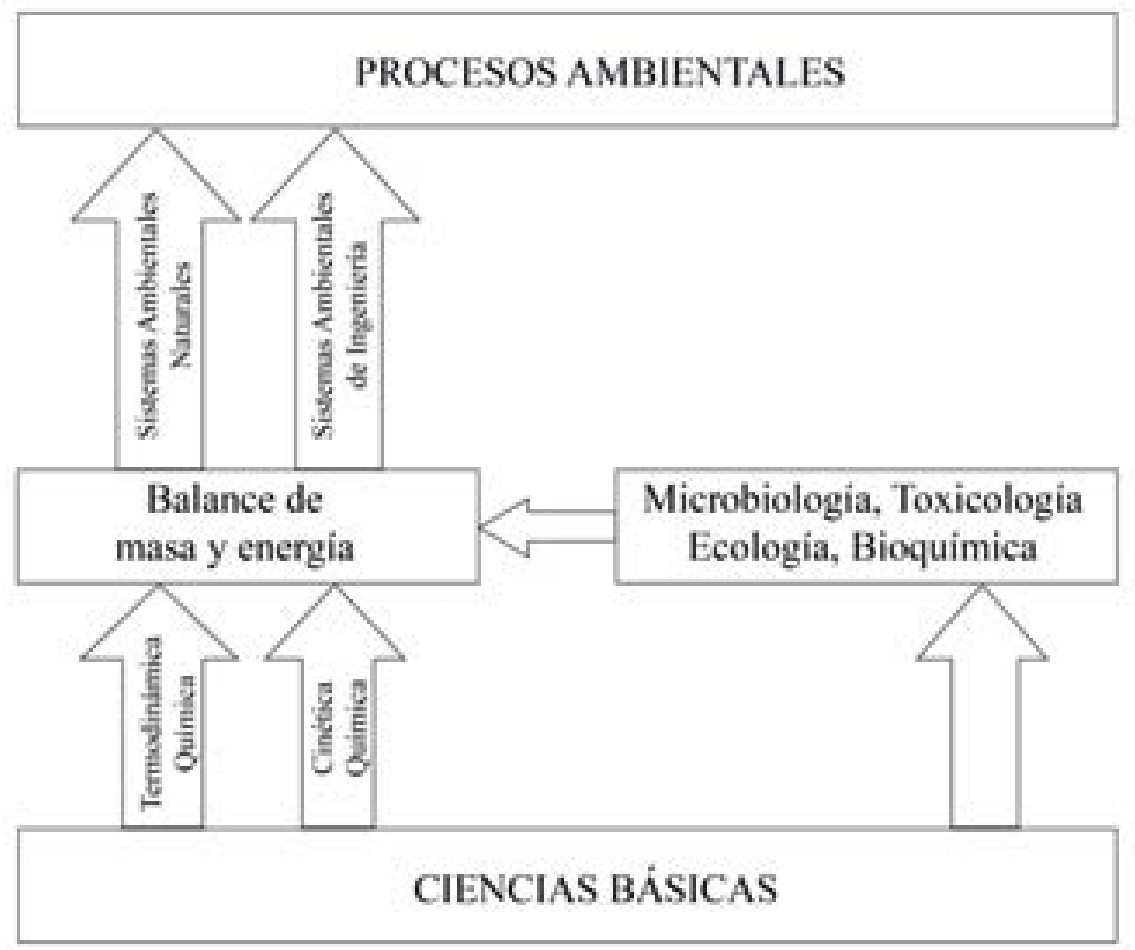

Figura 1. Pilares en la enseñanza de los procesos ambientales. Fuente: Adaptado de Valsaraj, 2000. 
La termodinámica química es la rama de la química relacionada con el estudio de las propiedades fisicoquímicas de una sustancia en los tres estados de la materia (sólido, líquido y gas). Asimismo, describe el potencial de la sustancia para moverse entre varias fases, como también su distribución final en las fases con que está en contacto (Valsaraj, 2000). Por su parte, la cinética química se remite a dos velocidades: la empleada por una sustancia para moverse entre fases y aquella con que reacciona en éstas (Schnoor, 1996). Ambas áreas, con el apoyo de la microbiología, la ecología, la toxicología y la bioquímica, definen la ecuación diferencial fundamental del balance de masa y energía. Dicha ecuación es la base para formular los modelos matemáticos de los procesos que se llevan a cabo en sistemas ambientales (Weber y DiGiano, 1996).

En general, los balances de masa que se plantean son regresiones polinómicas múltiples (lineales y no lineales), al igual que sistemas de distintas ecuaciones: algebraicas lineales o no lineales y diferenciales parciales, ordinarias y algebraicas. Todas pueden utilizarse para simulaciones dinámicas o para encontrar el valor de las variables en estado estacionario (Shachamy Cutlip, 1999).

Durante el curso de Procesos Ambientales, el estudiante plantea el balance de masa de acuerdo con el sistema en estudio y sus límites, soluciona las ecuaciones de forma manual para condiciones de estado estacionario o dinámicas y se plantea interrogantes acerca de la influencia de los parámetros de los modelos sobre las variables de estado. Una vez se tiene una comprensión adecuada de las bases teóricas, se incorporan herramientas computacionales que posibilitan la corroboración de los conceptos mediante la simulación numérica dinámica de los sistemas. Esta aplicación de modelos matemáticos y programas computacionales en el currículo de la Ingeniería Ambiental, es cada vez más frecuente como estrategia y herramienta de aprendizaje (Gujer, 2004; 2008).

De hecho, el avance en la informática ha generado la necesidad de introducir el uso de herramientas computacionales en el estudio y en la práctica de la Ingeniería Ambiental (Argent, 2004). En efecto, al ser instrumentos muy poderosos, los programas computacionales conllevan a una rápida y eficiente implementación de los modelos, así como a la realización de simulaciones complejas, que de otra forma, tomarían mucho tiempo. La simulación dinámica computarizada también ofrece una ventaja en términos de eficiencia sobre la solución manual, pues cuando el modelo matemático posee más de dos ecuaciones diferenciales, esta última se torna muy ineficiente.

Una cantidad considerable de conceptos teóricos puede abordarse en el aula mediante la combinación de ejercicios de modelos matemáticos de desarrollo manual y la aplicación de éstos en el computador (Gujer, 2008).

La formulación matemática de la realidad con base en modelos, ha sido presentada por Makinia (2010); Gujer (2008) y Nirmalakhandan (2002) como una gran ventaja, pues optimiza los costos por mediciones, favorece la toma de decisiones y evita la implementación de representaciones físicas que 
podrían resultar muy costosas. Estas herramientas, unidas a la solución manual de las ecuaciones diferenciales, propician una interacción dinámica entre estudiantes y profesores, al potenciar el análisis de escenarios de simulación a partir de la pregunta ¿"Qué pasa si..."? De esta forma, también es posible comparar los resultados entre la solución manual de los modelos y la salida de la simulación en el computador.

Debido a su gran variedad de funciones, estas herramientas representan un importante recurso para la implementación de modelos matemáticos, sencillos o complejos, en el estudio y la práctica de la Ingeniería Ambiental. Más aun, ante la diversificación e incremento de los problemas ambientales contemporáneos, el uso de métodos avanzados de monitoreo y de modelos matemáticos que permitan examinar los problemas, plantear escenarios y encontrar soluciones de forma rápida y eficiente, se ha convertido en un requerimiento de primer orden (Brenner,Shacham y Cutlip, 2005). Adicionalmente, es cada vez más importante conectar el estudio de la modelación matemática de los sistemas ambientales y la práctica de la Ingeniería Ambiental, Hug et al. (2009).

En la actualidad, existen muchos programas que pueden ser introducidos en la enseñanza y la práctica de la Ingeniería Ambiental, tales como ASIM (Gujer y Larsen, 1995) y AQUASIM (Reichert, 1995). Igualmente, se cuenta con herramientas de uso múltiple, entre ellas: Excel, Berkeley Madonna (Macey, Oster y Zahney, 2001); Excel (Gottfried, 2009), Stella, Ithink, (Nirmalakhandan, Egemen y Edwards, 1998; Deatony Winebrake, 2000; Nirmalakhandan, 2002; Ford, 2009), Matlab (Martinez y Rodriguez, 2005, Gil-Rodríguez, 2005), y POLYMATH, (Brenner, Shacham y Cutlip, 2005). Otras herramientas, entre ellas GPS-X 6.0, WEST y BIOWIN (Makinia, 2010; Grady, Daigger, Love y Filipe, 2011), son usadas como simuladores en cursos de procesos biológicos y diseño de plantas de tratamiento de aguas residuales.

Una de las características más importantes de estos programas y simuladores es su interface amigable, la cual permite una rápida conexión entre los conceptos teóricos y la salida de los modelos. Esta cualidad facilita el desarrollo de las ecuaciones de balance de masa, así el estudiante no cuente con un amplio conocimiento de lenguajes de programación (Nirmalakhandan, Egemen y Edwards, 1998).

Ahora bien, durante el desarrollo del curso de Procesos Ambientales debe tenerse especial cuidado, pues como lo menciona Gujer (2004), los estudiantes tienden a prestar más atención a la implementación del código computacional o a la simulación que a los fundamentos teóricos de los ejercicios. Por lo tanto, para aplicar las herramientas se sugiere una planificación adecuada de los contenidos, en aras de armonizar la comunicación de los componentes teóricos con el desarrollo de ejercicios manuales que luego sean trasladados al programa computacional. De tal forma, el estudiante puede corroborar sus planteamientos conceptuales y enfrentar con éxito ejercicios más complejos. En este sentido, un mensaje importante al comienzo del curso es el siguiente: el computador y el programa son de gran ayuda, pero si no se ha alcanzado un nivel adecuado en la comprensión de la teoría básica, las aplicaciones efectuadas no serán exitosas. 
En el caso de la Universidad de Boyacá, la clase tiene lugar en el centro de informática y cada alumno dispone de un computador para realizar las simulaciones. El profesor permanece atento a la ejecución de los ejercicios y presenta los resultados al tiempo con los estudiantes. El producto de una simulación se enfrenta con uno o varios conceptos teóricos, por consiguiente, se induce el análisis de lo obtenido. Este procedimiento reviste una notoria relevancia, pues suele creerse que cuando la herramienta computacional proporciona una respuesta, el resultado es siempre correcto. Mediante tal sistema de aprendizaje, se busca que el alumno se concentre más en el tópico específico abordado y no en los detalles técnicos del programa empleado. Cabe señalar que la herramienta seleccionada debe ser de fácil comprensión y ha de ofrecer los resultados en forma gráfica para facilitar su interpretación.

En la Figura 2 se presenta el curso de Procesos Ambientales del programa de Ingeniería Ambiental de la Universidad de Boyacá. A la izquierda se observa la secuencia de los contenidos básicos, y en el bloque de la derecha, aquellos más avanzados. El aprendizaje de todos los temas puede acompañarse de una herramienta como las mencionadas aun cuando se abarquen tópicos más avanzados, entre ellos, la estimación de los parámetros del modelo, el análisis de sensibilidad para evaluar la importancia de éstos y el análisis de incertidumbre al considerar modelos de carácter estocástico (Gujer, 2008) ${ }^{1}$.

\footnotetext{
${ }^{1}$ Ejemplos de la aplicación de herramientas computacionales en todos los contenidos de un curso de Procesos Ambientales, pueden encontrarse en Bijlsma y Hooijmans (1996), Gujer (2000), Gujer (2004), Gujer (2008), Ingham et.al. (2008). Además, para el caso de modelación de procesos biológicos, en TU-Delft, IHE-Delft (2002).
} 


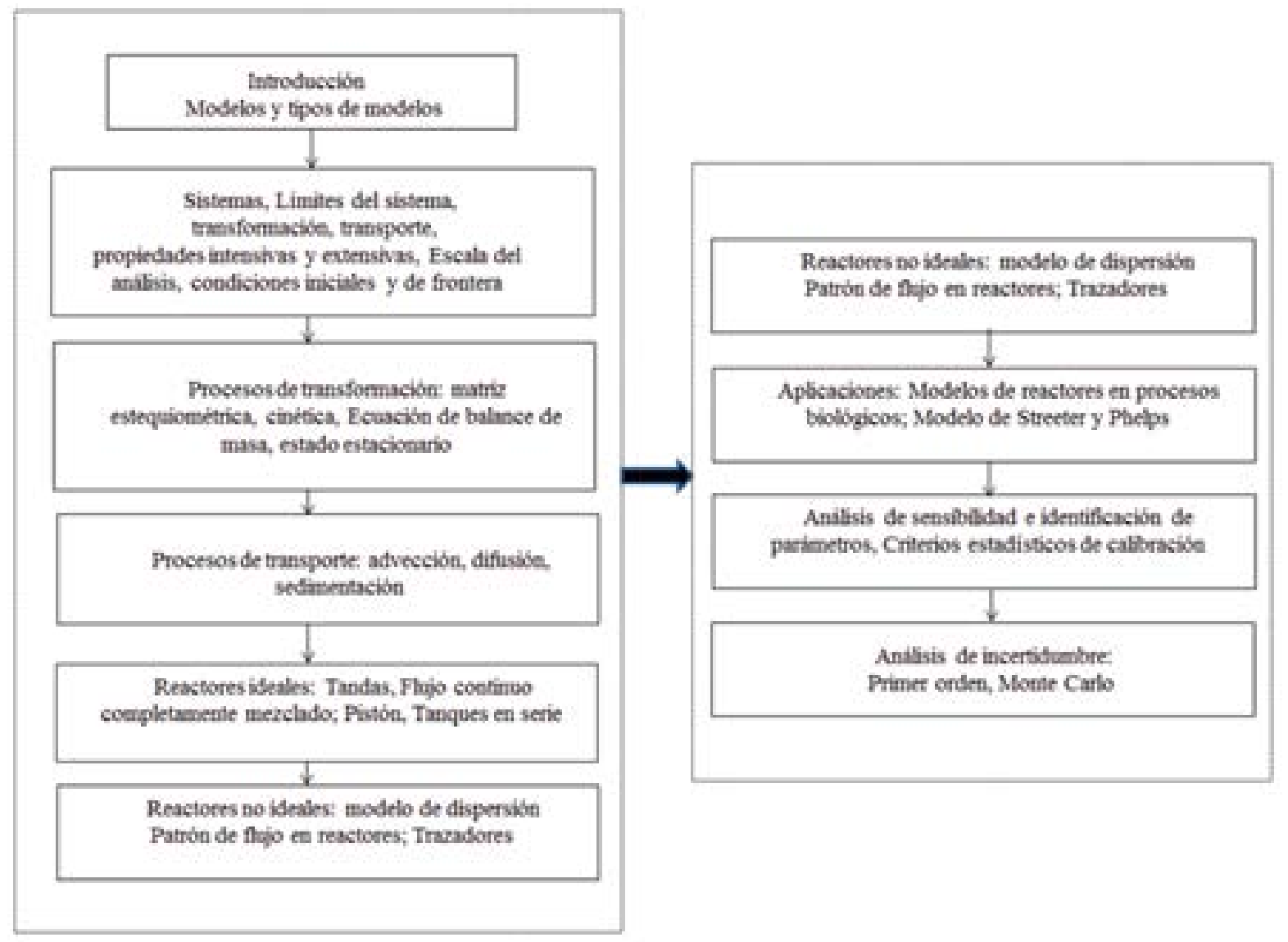

Figura 2. Contenido del curso de Procesos Ambientales. Fuente: Adaptado de Gujer, 2008.

En este artículo se presenta un ejemplo implementado en el curso de Procesos Ambientales, cuando los estudiantes se encuentran en la fase de aplicaciones. La metodología del curso se sustenta en los conceptos del aprendizaje basado en problemas, cuyo fundamento señala que el estudiante necesita comprender las bases teóricas para abordar un problema e implementar luego ese conocimiento en la formulación de soluciones (Egemen, Edwards y Nirmalakhandan, 1998). Un punto a considerar es el manifestado por Felder (1988): si los estudiantes son pasivos y tienen poco tiempo para reflexionar, es primordial capturar su atención e incrementar su participación en clase. Al respecto, los modelos matemáticos y la simulación por computador constituyen herramientas amigables y visualmente atractivas, de ahí su efectividad para lograr dichos propósitos. 


\section{METODOLOGÍA}

En este curso se adoptó Berkeley Madonna 8.3.18 (BM), un solucionador de ecuaciones diferenciales de propósito múltiple (Macey, Oster y Zahney, 2001) Entre sus ventajas se cuentan la facilidad de lectura de su código, su precio económico y su amplia disponibilidad. Incluso en su versión de demostración, es posible utilizarlo en la mayoría de contenidos (Gujer, 2008).

El ejercicio planteado como ejemplo es la simulación dinámica de un reactor biológico aerobio de flujo continuo y mezcla completa, con sedimentador secundario y recirculación. Este mecanismo puede ser usado en el tratamiento de aguas residuales por medio de lodos activados, ya sean convencionales o por aireación extendida. Así, el objetivo del trabajo es: identificar, gracias a un modelo matemático implementado en una herramienta computacional, las diferencias existentes entre la tecnología de lodos activados convencionales y la de lodos activados por aireación extendida.

Con anterioridad a la realización del ejercicio, los estudiantes reciben información acerca de las características de cada tecnología. Este soporte teórico se encuentra en los textos más reconocidos relacionados con el tratamiento de aguas residuales, como es el caso de Metcalf y Eddy (2003). La diferencia sustancial entre las tecnologías es la edad de los lodos $\left(\theta_{c}\right)$, cuyo valor ronda los 5 días para los activados convencionales, mientras que para los activados por aireación extendida, es de 20 días.

Con el empleo de un modelo matemático del proceso biológico, elaborado en $\mathrm{BM}$, se espera resolver las siguientes preguntas:

- ¿Cuáles son las implicaciones de operar los sistemas con los valores indicados para la edad del lodo?

- De acuerdo con dicha edad, ¿cuáles son las características de los procesos que deben considerarse en lodos activados convencionales y en lodos activados por aireación extendida?

La primera actividad consiste en identificar los componentes del sistema a estudiar, al igual que los límites a considerar en el balance de masa. El estudiante elabora una matriz como la que se observa en la Tabla 1. 


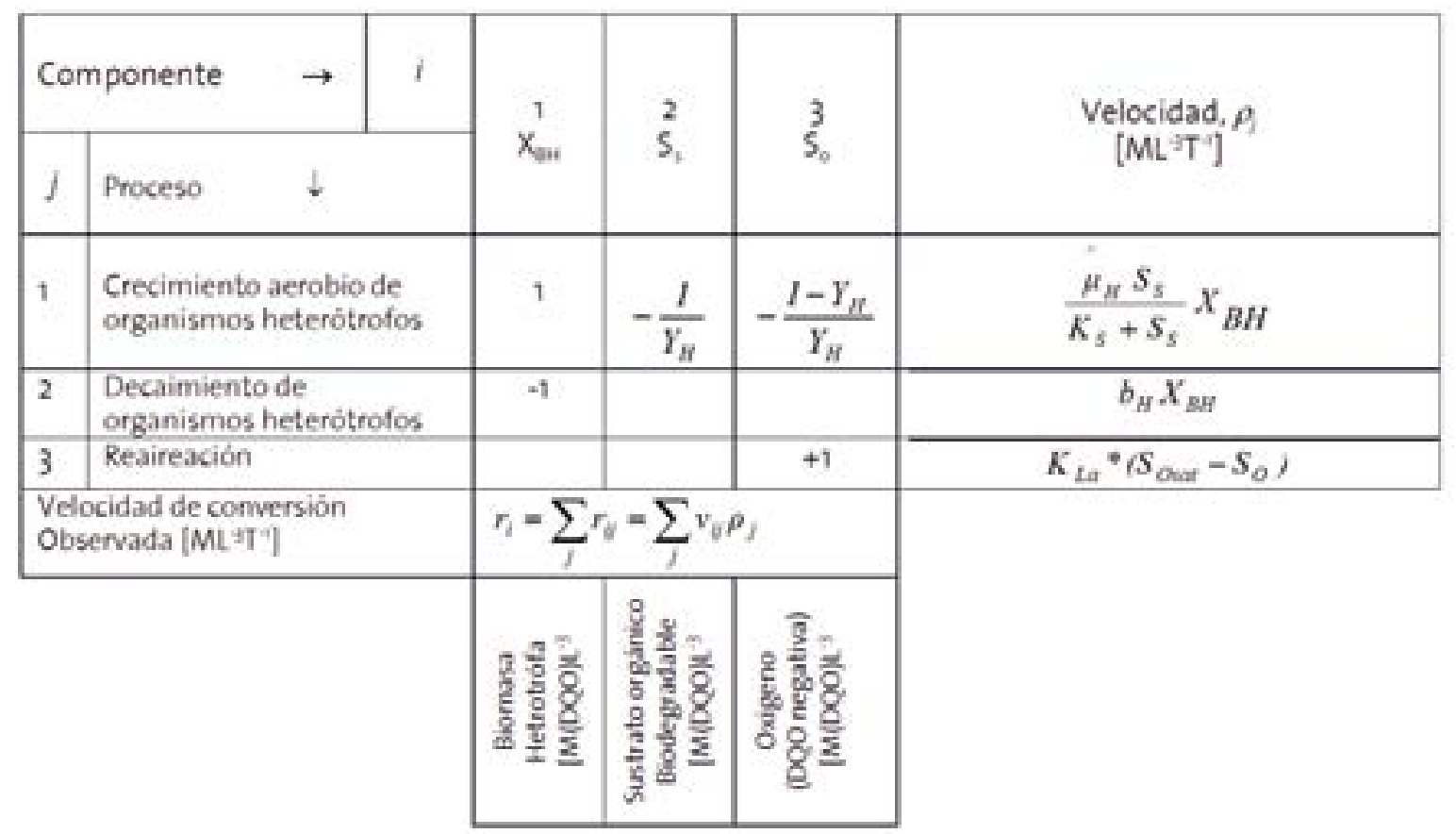

Tabla 1. Modelo biocinético empleado en el ejemplo. Fuente: Modificado de Henze et al., 2000.

En la Tabla 1:

$\mu_{H}$ : Constante de crecimiento específico máxima $\left[\mathrm{T}^{-1}\right]$

$\mathrm{K}_{\mathrm{s}}$ : Constante de saturación $\left[\mathrm{M} / \mathrm{L}^{3}\right]$

$\mathrm{b}_{\mathrm{H}}$ : Constante de decaimiento $\left[\mathrm{T}^{-1}\right]$

$\mathrm{S}_{\text {Osat }}:$ Concentración de saturación del Oxígeno $\left[\mathrm{M} / \mathrm{L}^{3}\right]$

$\mathrm{K}_{\mathrm{La}}$ : Tasa de transferencia de Oxígeno $\left[\mathrm{T}^{-1}\right]$

$\mathrm{Y}_{\mathrm{H}}$ : Coeficiente de producción de los organismos heterótrofos [P biomasa-DQO/P sustrato DQO]

La matriz es el modelo biocinético manejado en el ejercicio. Los conceptos del modelo biocinético han sido previamente estudiados en los cursos de Química Ambiental. Para este caso el modelo biocinético está basado en el modelo ASM 1 (Activated Sludge Model 1), (Henze et al., 2000). Este modelo fue adoptado por la International Water Association (IWA) con base en el trabajo de Petersen (1965) y los aportes de Grau et al. (1982). Su aplicación ha sido ampliada a sistemas ambientales naturales como ríos (Shanahan et al., 2001) 
Para el caso aquí descrito se incorpora una simplificación del modelo ASM 1, la cual incluye tres procesos: crecimiento aerobio de organismos heterótrofos, decaimiento de éstos y reaireación. En la matriz que se construye, se distinguen varios elementos: procesos (j), constituyentes (i), coeficientes estequiométricos $\left(\cup_{\mathrm{ij}}\right)$ y velocidad de los procesos $\left(\rho_{\mathrm{i}}\right)$. En el modelo biocinético resumido, presentado en la Tabla 1, se asume que el valor de la constante de crecimiento específico de los organismos heterótrofos $\left(\mu_{\mathrm{H}}\right)$ se limita solo por el sustrato orgánico rápidamente biodegradable $\left(\mathrm{S}_{\mathrm{s}}\right)$.

Según el modelo biocinético, la velocidad de transformación de un constituyente específico corresponde a la sumatoria del producto de los coeficientes estequiométricos por la cinética del proceso. Es pertinente indicar que en un sistema, la concentración de un constituyente puede verse afectada por un número cambiante de procesos. Así, dicha velocidad está dada por:

$r_{i}=\sum_{j} r_{j}=\sum_{j} i_{j} \tilde{n}_{j}$

Un beneficio de la matriz que representa al modelo biocinético, es su rapidez para reconocer el destino de cada constituyente, condición de gran ayuda al momento de plantear el balance de masa (Henze et al., 2000; Gujer y Henze, 1991). La expresión de este balance para un constituyente en un límite de sistema definido es

Entrada- Salida + Reacción $=$ Acumulación

Por ejemplo, para el sistema del ejercicio aquí tratado, esta expresión incluye los flujos advectivos de entrada y salida, lo mismo que la reacción representada por la ecuación 1 . Tal reacción está conformada por la suma del producto de los coeficientes estequiométricos vij, y la cinética de la reacción $\rho \mathrm{j}$ del componente i, considerado en el balance de masa (Henze et al., 2000) 


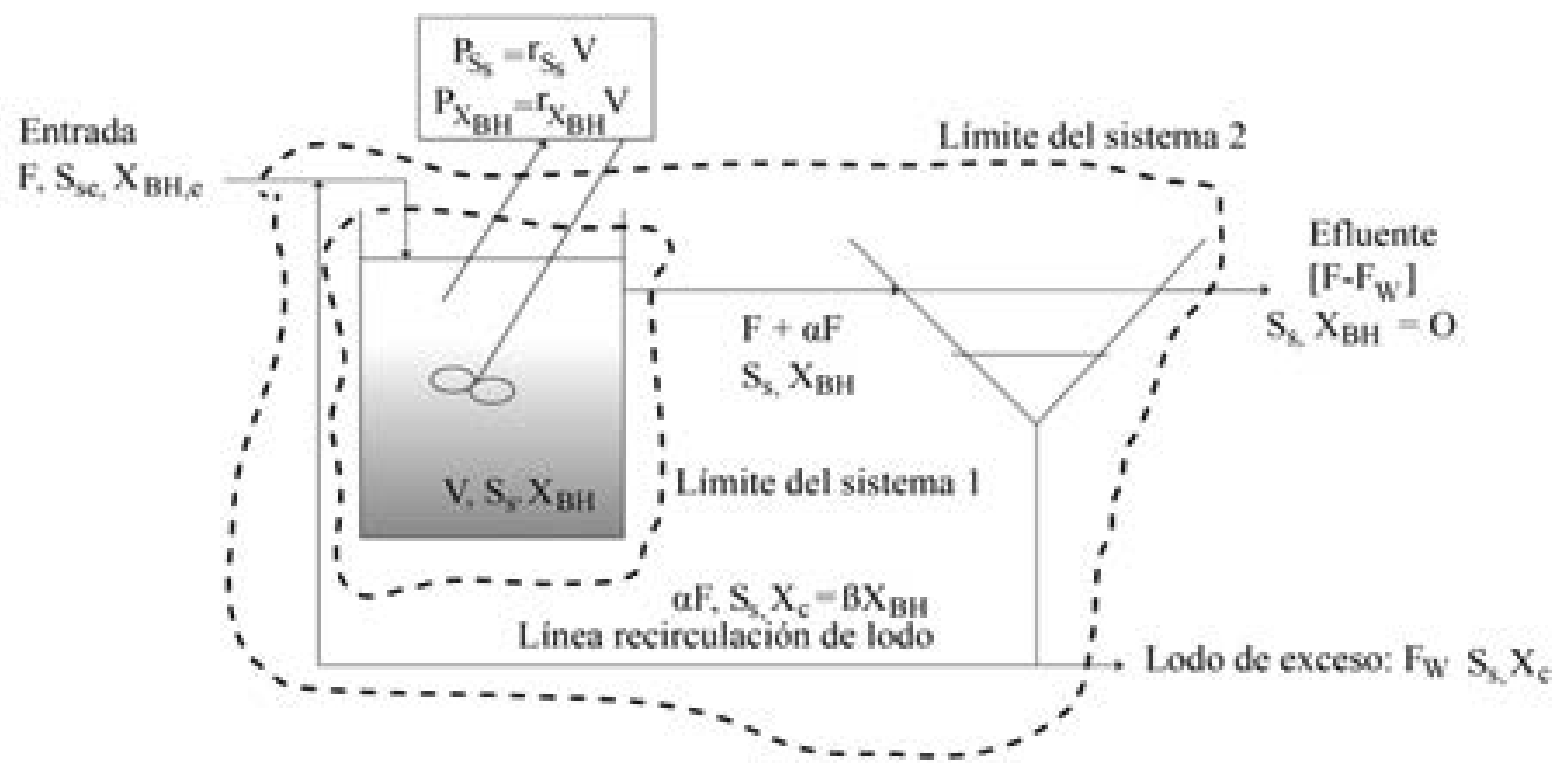

Figura 3. Reactor biológico aerobio.

Con base en el modelo biocinético, en los principios de la formulación del balance de masa y en el sistema presentado en la Figura 3, el estudiante plantea los balances de masa teniendo en cuenta el límite del sistema 1 y el del sistema 2, el volumen del tanque de aireación y del sedimentador secundario son constantes.

Balance de masa para el sustrato orgánico biodegradable, $\mathrm{S}_{\mathrm{s}}$, en el reactor:

$$
V \frac{d\left(S_{s}\right)}{d t}=F S_{s}+\alpha F S_{s}-(I+\alpha) F S_{s} \cdot\left(\frac{I}{Y_{N}}\right) \mu_{H} X_{\text {sw }} V
$$

De la ecuación anterior:

$$
V \frac{d\left(S_{s}\right)}{d t}=F S_{S}-F S_{s} \cdot\left(\frac{I}{Y_{H}}\right) \mu_{H} X_{R H} V
$$


Donde:

V: Volumen del tanque de aireación $\left[\mathrm{L}^{3}\right]$

F: Flujo que entra al reactor $\left[\mathrm{L}^{3} / \mathrm{T}\right]$

El balance de masa para la biomasa heterótrofa, $\mathrm{X}_{\mathrm{BH}}$, en el reactor asumiendo que la concentración de oxígeno en la línea de recirculación de lodo es cero, es:

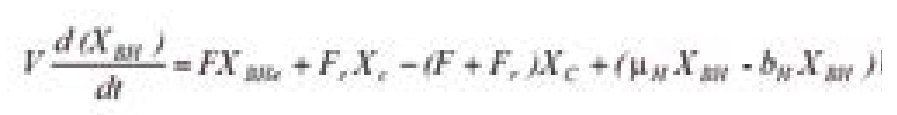

\section{Donde:}

$\mathrm{F}_{\mathrm{r}}$ : Flujo de recirculación $\left[\mathrm{L}^{3} / \mathrm{T}\right]$

$\mathrm{X}_{\mathrm{c}}^{\mathrm{r}}$ : Biomasa en la línea de recirculación [M Biomasa DQO $\left./ \mathrm{m}^{3}\right]$

El balance de masa en el sedimentador secundario, $X_{C}$, sin considerar reacción en el sedimentador, es:

$$
\frac{d X_{C}}{d t}=\left(\frac{F_{r}+F}{V_{t}}\right) \cdot X_{B R t} \cdot\left(\frac{F_{z}+F_{w}}{V_{t}}\right) \cdot X_{c}
$$

Donde:

$\mathrm{V}_{\mathrm{s}}$ : Volumen del sedimentador secundario $\left[\mathrm{L}^{3}\right]$

$\mathrm{F}_{\mathrm{w}}$ : Flujo de lodo de exceso $\left[\mathrm{L}^{3} / \mathrm{T}\right]$

El balance de masa en el tanque de aireación para el Oxígeno, $\mathrm{S}_{\mathrm{O}}$, es:

$$
\frac{d S_{o}}{d t}=\frac{F * S_{o *}}{\mathrm{~V}}-\frac{F^{*} S_{O}}{\mathrm{~V}}+r_{\text {so }}
$$

Del modelo biocinético, la tasa de utilización de Oxígeno, $\mathrm{r}_{\text {so }}$, es:

$$
r_{S O}=-\left(\left(1-Y_{H}\right) / Y_{H}\right) * \mu_{H} * X_{B H}+K_{L a} *\left(S_{O s a t}-S_{S}\right)
$$


El modelo de Jacques Monod expresa la relación entre el sustrato limitante para el caso $\mathrm{S}_{\mathrm{s}}$, y la constante de crecimiento específico, así:

$$
\mu_{H}=\frac{\mu_{N} S_{S}}{K_{S}+S_{S}}
$$

En estas ecuaciones diferenciales, que representan un balance de masa para la variación de las variables de estado de interés, se identifican los componentes teóricos aprendidos en los cursos de ciencias naturales y formación básica, como son: la química, manifestada por la velocidad de la reacción, el carácter rédox de la reacción biológica y su estequiometría; las matemáticas, identificadas por ecuaciones diferenciales ordinarias; la primera y la segunda ley de la termodinámica, que indican la conservación de la masa, la energía y la dirección de las posibles transformaciones; la física, aplicada en la sedimentación del lodo biológico; la microbiología, contemplada en la implementación del modelo de Jacques Monod; y los conceptos referentes a reactores ideales, presentes en el reactor de flujo continuo y mezcla completa, correspondiente al tanque de aireación.

El concepto de la edad del lodo, estudiado previamente en clase, se expresa de forma general como:

Edad del lodo $=\frac{\text { Masa de lodoen el reactor }}{\text { Cargade lodo de exceso }}$

En el campo del diseño y control de procesos biológicos de tratamiento, la edad del lodo es un parámetro particularmente útil, debido a su relación con la tasa de crecimiento biológico y a su relativa facilidad de comprensión (Lawrence y Mc Carty, 1970).

Para el sistema en estudio, esta edad es:

$$
\theta_{C}=\frac{X_{B H} * V}{F_{z} * X_{c}}
$$

El concepto ya ha sido discutido en clase y se ha definido para varias configuraciones de un sistema de tratamiento de aguas residuales. Con base en lo anterior, se determina que el caudal de lodo de exceso $\left(\mathrm{F}_{\mathrm{w}}\right)$ será la variable a usar para operar el sistema y reconocer la influencia de la variación de la edad del lodo sobre las variables de estado $\mathrm{S}_{\mathrm{s}}, \mathrm{X}_{\mathrm{BH}}$ y $\mathrm{S}_{\mathrm{O}}$. 
La pregunta formulada a manera de escenario es:

¿Qué pasa si variamos el caudal de lodo de exceso?

A continuación, el estudiante elabora un código, escrito en BM, que puede ser empleado para la simulación dinámica propuesta en el ejemplo. Los valores de las constantes cinéticas del modelo fueron tomadas de Henze et al. (2000).

\section{METHOD RK4 \\ STARTTIME $=0$ \\ STOPTIME $=4$ \\ $\mathrm{DT}=0.02$}

\{Reactor biológico aerobio de flujo continuo y mezcla completa con recirculación\}

\{Constantes\}

$\operatorname{Mumax}=6$

$\mathrm{Ks}=20$

$\mathrm{bH}=0.62$

$\mathrm{YH}=0.71$

Sse $=250$

$\mathrm{SOe}=0.3$

$\mathrm{F}=2000$

$\mathrm{Fr}=200$

$\mathrm{Fw}=300$

$\mathrm{V}=600$

$\mathrm{Vs}=35$

$\mathrm{KLa}=490$

SOsat $=7.02$ \{constante máxima de crecimiento específico, d-1\} \{constante de saturación, g DQO/m^ 3$\}$

$\left\{\right.$ Constante de decaimiento, $\left.\mathrm{d}^{\wedge}-1\right\}$

$\{\mathrm{g}$ Biomasa-DQO/g sustrato DQO $\}$

\{Sustrato afluente, $\left.\mathrm{g} \mathrm{DQO} / \mathrm{m}^{\wedge} 3\right\}$

\{Concentración de Oxígeno disuelto entrando\}

\{caudal entrando al reactor, $\left.\mathrm{m}^{\wedge} 3 / \mathrm{d}\right\}$

\{caudal de recirculación, $\mathrm{m}^{\wedge} 3 / \mathrm{d}$ \}

\{Caudal lodo de exceso, $\mathrm{m}^{\wedge} 3 / \mathrm{d}$ \}

\{Volumen del tanque de aireación, $\mathrm{m}^{\wedge} 3$ \}

\{Volumen del sedimentador secundario, $\mathrm{m}^{\wedge} 3$ \}

$\left\{\right.$ Coeficiente de transferencia de Oxígeno, $\left.\mathrm{d}^{\wedge}-1\right\}$

$\{$ concentración de saturación de Oxígeno, g DQO/m^^3\}

\{Condiciones iniciales

INIT Ss $=250$

INIT XBH $=1000$

INIT Xc $=500$

INIT $\mathrm{SO}=2$ 
\{Balances de masa\}

$\mathrm{d} / \mathrm{dt}(\mathrm{Ss})=\mathrm{F} * \mathrm{Sse} / \mathrm{V}-\mathrm{F} * \mathrm{Ss} / \mathrm{V}-(\mathrm{Mu} / \mathrm{YH})^{*} \mathrm{XBH} \quad\{$ Sustrato de organismos heterótrofos, g DQO/m^3 3$\}$

\{Balance de masa de biomasa heterótrofa\}

$\mathrm{d} / \mathrm{dt}(\mathrm{XBH})=(\mathrm{Fr} / \mathrm{V}) * \mathrm{Xc}-((\mathrm{Fr}+\mathrm{F}) / \mathrm{V}) * \mathrm{XBH}+\mathrm{Mu}^{*} \mathrm{XBH}-\mathrm{bH} * \mathrm{XBH} \quad$ \{Biomasa heterótrofa, g DQO/m^3\}

$\mathrm{Mu}=$ Mumax* $\mathrm{Ss} /(\mathrm{Ks}+\mathrm{Ss}) \quad\left\{\right.$ Monod, constante de crecimiento específico, $\left.\mathrm{d}^{\wedge}-1\right\}$

\{Balance de masa de biomasa de recirculación Sedimentador secundario\}

$\mathrm{d} / \mathrm{dt}(\mathrm{Xc})=((\mathrm{Fr}+\mathrm{F}) / \mathrm{Vs})^{*} \mathrm{XBH}-((\mathrm{Fr}+\mathrm{Fw}) / \mathrm{Ns})^{*} \mathrm{Xc}$

$\{$ Biomasa heterótrofa en la línea de recirculación, g Biomasa-DQO/m^3\}

\{Balance de masa de Oxígeno Disuelto. Tanque de aireación\}

$\mathrm{d} / \mathrm{dt}(\mathrm{SO})=-(\mathrm{F} /)^{*} \mathrm{SO}+\mathrm{F}^{*} \mathrm{SOe} / \mathrm{N}+\mathrm{rSO}$

\{Oxígeno disuelto, g DQO/m^3\}

$\mathrm{rSO}=-((1-\mathrm{YH}) / \mathrm{YH}) * \mathrm{Mu}^{*} \mathrm{XBH}+\mathrm{KLa}^{*}\left(\mathrm{SO}\right.$ at-SO) $\left\{\right.$ tasa de consumo de Oxígeno disuelto, $\left.\mathrm{g} \mathrm{DQO} / \mathrm{m}^{\wedge} 3 * \mathrm{~d}\right\}$

$\mathrm{Edad}=\left(\mathrm{V}^{*} \mathrm{XBH}\right) /\left(\mathrm{Fw}^{*} \mathrm{Xc}\right)$

\{Edad del lodo, días\}

En el programa computacional escrito en BM, puede observarse la relativa facilidad de lectura de su código, una vez que el estudiante ha elaborado los balances de masa con base en el sistema estudiado. Cuando se termina de escribir dicho código, se enfatiza en su relación con el modelo matemático, procedimiento en el cual se identifican las variables de estado del modelo, las condiciones iniciales de estas variables, los balances de masa, los parámetros representados como constantes y el tiempo de la simulación. Asimismo, se discute la posibilidad de emplear distintos métodos numéricos para solucionar las ecuaciones diferenciales.

\section{RESULTADOS Y DISCUSIÓN}

Los resultados se presentan en gráficos, que representan la variación de las variables de estado del modelo. En este caso, se aprovechan algunas características de los programas computacionales mencionados, como son la posibilidad de efectuar simulaciones simultáneas solo con cambiar algunos parámetros y sin salir del ambiente de simulación, e igualmente, el potencial para colocar el resultado en un mismo gráfico con el fin de realizar comparaciones de forma rápida. Cuando se llega a este punto, se responde la siguiente pregunta: ¿lo obtenido está de acuerdo con los principios teóricos discutidos en clase?

En el ejercicio en cuestión, la pregunta formulada se refiere a la influencia del caudal de lodo de exceso sobre la eficiencia del proceso para transformación de la materia orgánica biodegradable. Ante este interrogante, el alumno corre la simulación para varios caudales de lodo de exceso. 


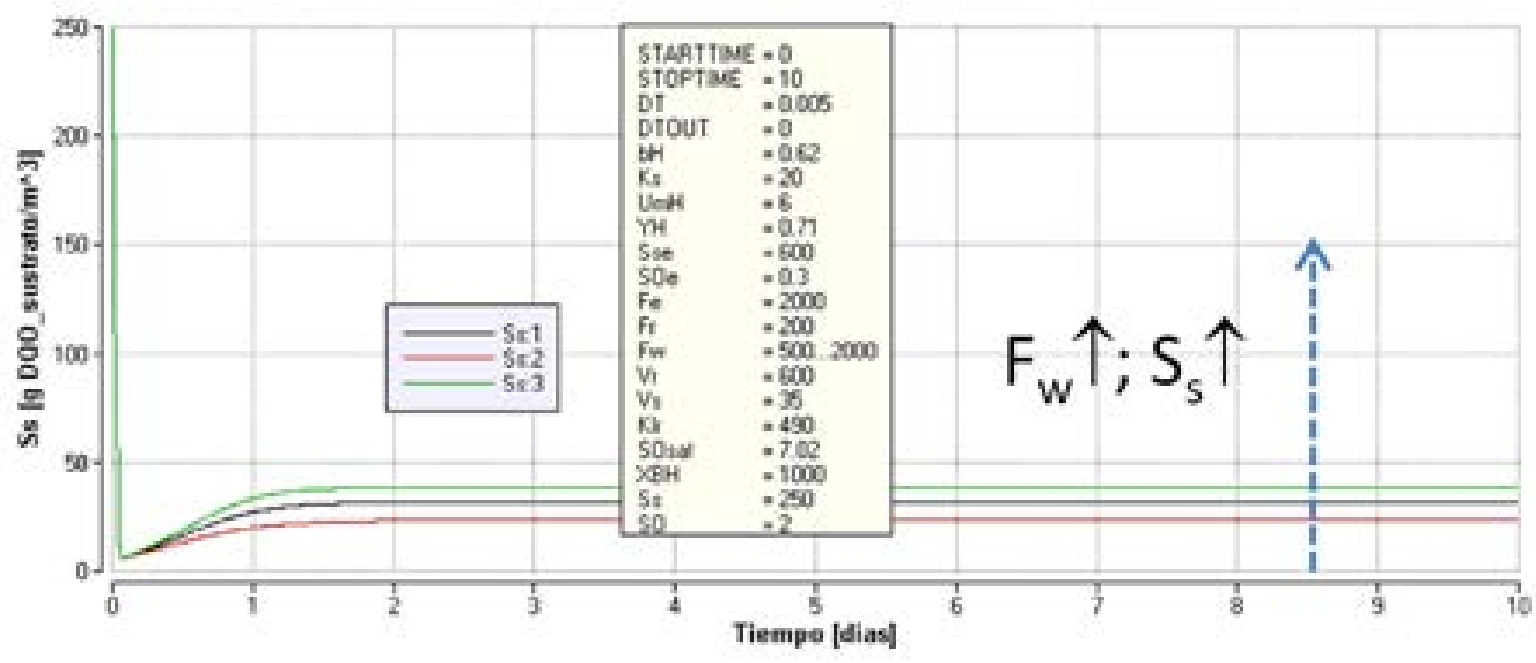

Figura 4. Relación entre la edad del lodo, la concentración de sustrato orgánico y el caudal de lodo de exceso (F $F_{w}: 500,1000,2000$ m³/d).

Lo revelado por la simulación en la Figura 4 debe corresponder con un concepto teórico: el aumento en el caudal de lodo de exceso disminuye la edad del lodo. Al decrecer ésta, el valor de la constante de crecimiento específico $\left(\mu_{\mathrm{H}}\right)$ también se reduce, pues previamente se ha desarrollado un balance de masa para la biomasa heterótrofa, en estado estacionario, en el que se ha llegado a la expresión:

$$
\frac{I}{\theta_{C}}=\mu_{H}-b_{H}
$$

Si el valor de $\mu_{\mathrm{H}}$ se aminora, el modelo de Monod sugiere que el valor de la concentración de sustrato limitante, $S_{s}$, presente en el reactor, se hace igualmente menor. 


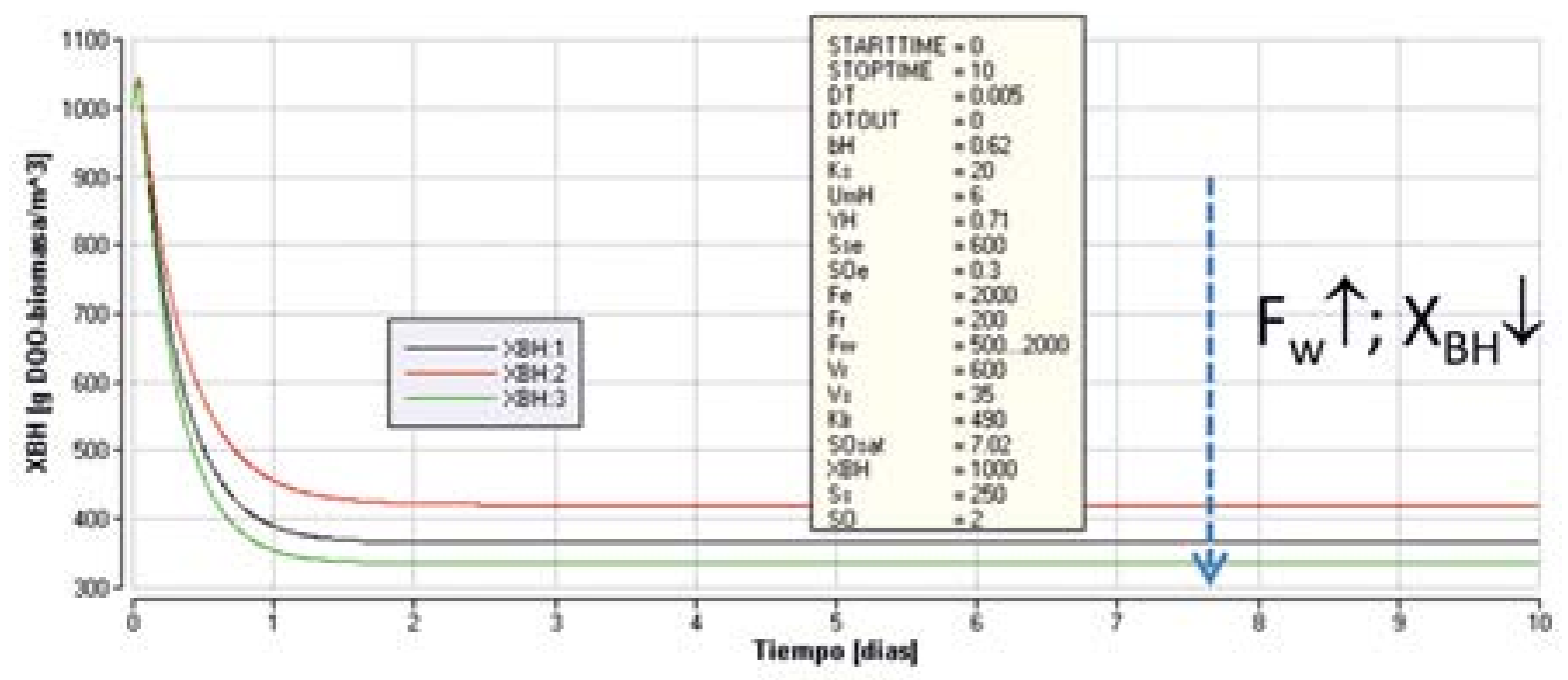

Figura 5. Relación entre la edad del lodo, la biomasa heterótrofa, y el caudal de lodo de exceso ( $F_{w}$ : 500, 1000, $\left.2000 \mathrm{~m}^{3} / \mathrm{d}\right)$

El aumento en el valor de $\mathrm{F}_{\mathrm{w}}$ debe producir una disminución en el valor de la concentración de biomasa en el tanque de aireación. Esto se debe a la relación directamente proporcional entre la edad del lodo y la concentración de biomasa en el reactor, como también a su relación indirectamente proporcional con el valor de $\mathrm{F}_{\mathrm{w}}$ (ver Figura 5).

En la Figura 6 se aprecia la relación definida entre el valor de $\mathrm{F}_{\mathrm{w}}$ y la concentración de Oxígeno Disuelto $\left(\mathrm{S}_{\mathrm{o}}\right)$ en el reactor. Un aumento de $\mathrm{F}_{\mathrm{w}}$ causa una reducción de la edad del lodo, y como se demostró anteriormente, ante una reducción en la concentración de biomasa heterótrofa en el tanque de aireación, se requiere una menor cantidad de Oxígeno. 


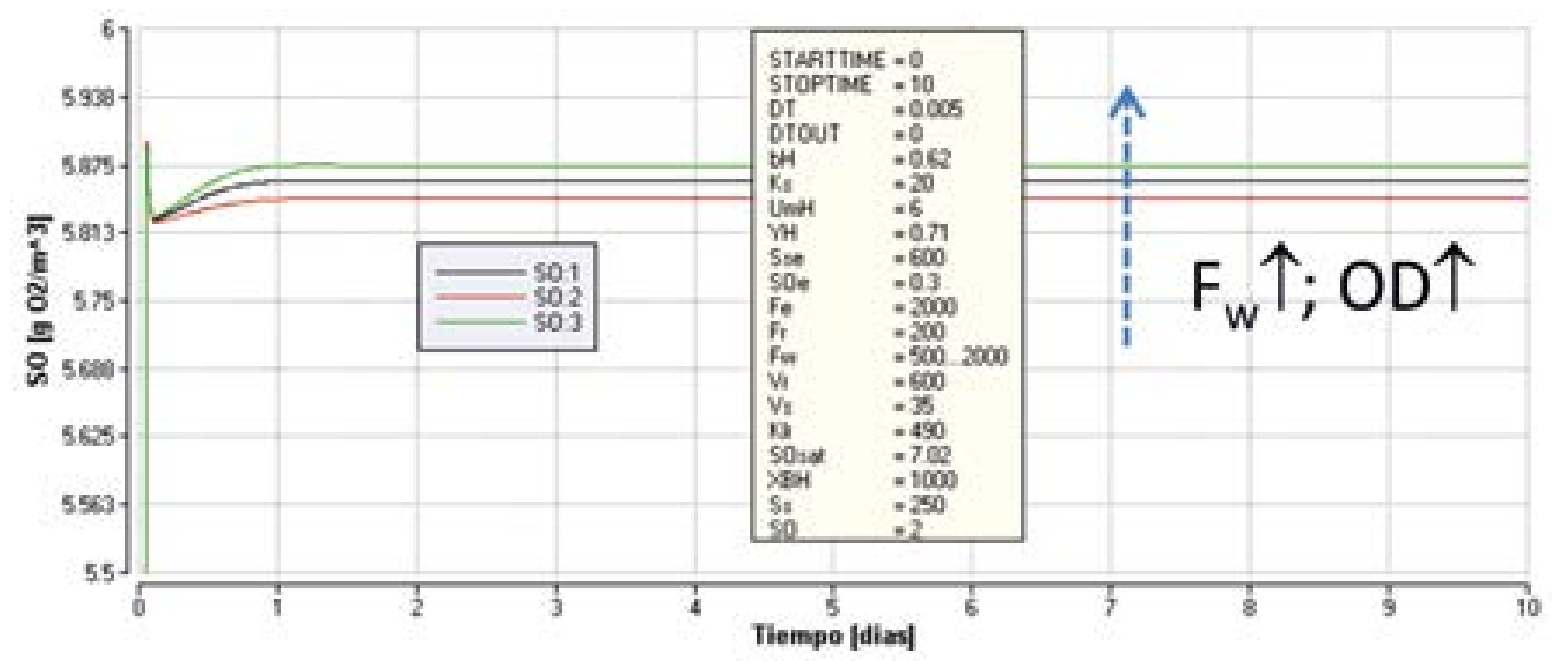

Figura 6. Relación entre la edad del lodo, la concentración de oxígeno disuelto y el caudal de lodo de exceso ( $\left.F_{w}: 500,1000,2000 \mathrm{~m}^{3} / \mathrm{d}\right)$

La Figura 7 refleja la relación indirectamente proporcional entre el valor de $\mathrm{F}_{\mathrm{w}} \mathrm{y}$ la edad del lodo. El estudiante adquiere el anterior concepto cuando plantea la expresión para calcular la edad del lodo, según sea la localización de la evacuación del lodo de exceso en el sistema.

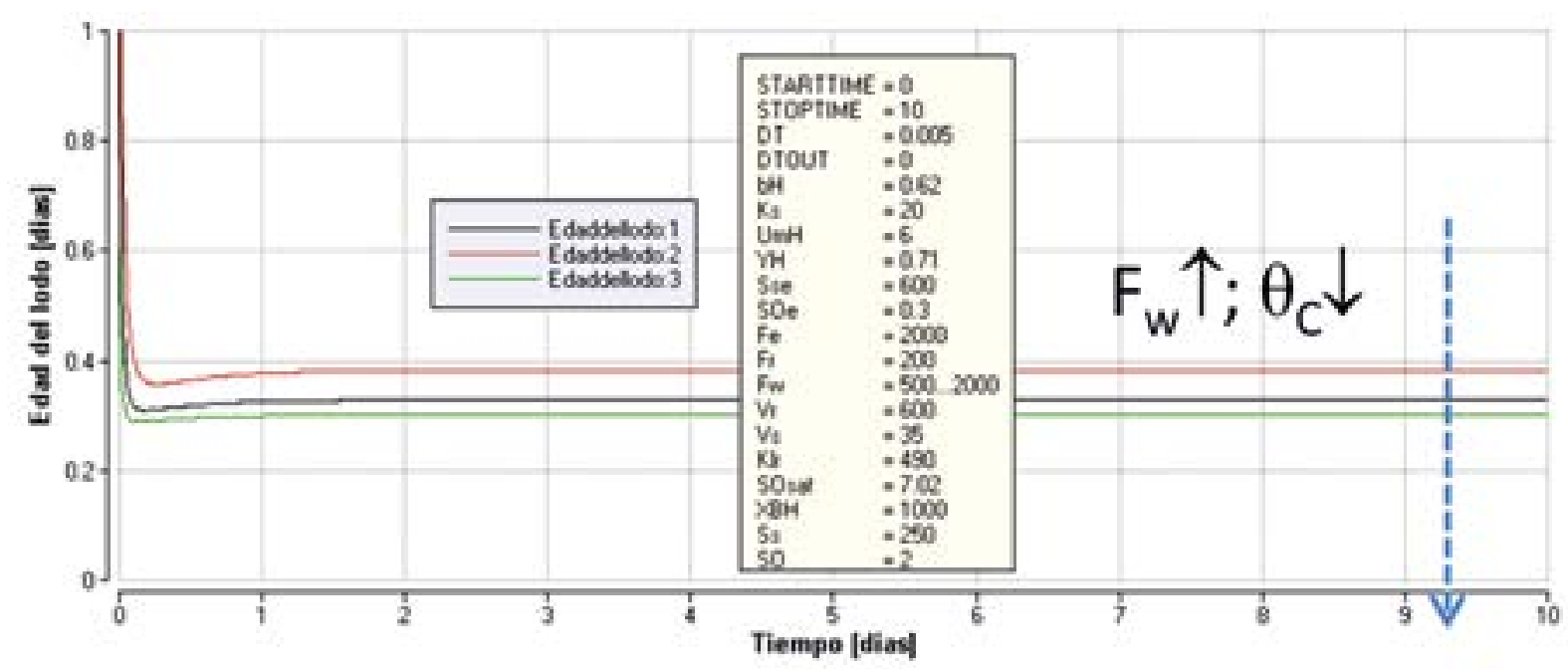

Figura 7. Relación entre la edad del lodo, y el caudal de lodo de exceso $\left(F_{w} ; 500,1000,2000 \mathrm{~m}^{3} / \mathrm{d}\right)$ 
Con base en los resultados obtenidos luego de correr el modelo, como también en la información proporcionada por la Tabla 2, es posible concluir que la aireación extendida, al operar con una edad del lodo más elevada, produce mayor eficiencia en la remoción de materia orgánica, en comparación con los lodos activados convencionales. Una consecuencia desventajosa de tal comportamiento es la elevada concentración de biomasa en el reactor, pues esta circunstancia, al hacer necesario un suministro de Oxígeno más alto, produce un incremento en los costos de energía.

Por el contrario, en los procesos biológicos cuya ejecución requiere una alta edad del lodo, el lodo de exceso se torna más estable, de ahí que para su disposición final no se precise una estabilización con procesos de digestión, pues basta con utilizar sistemas de tratamiento como el espesador, y posteriormente los lechos de secado o unidades como el filtro prensa. Lo anterior representa una ventaja, ya que en sistemas de tratamiento con baja edad del lodo, este material es muy crudo y debe ser estabilizado, por medio de digestión aerobia o anaerobia previa, para su disposición final.

\begin{tabular}{|c|c|}
\hline Ventajas & Desventajas \\
\hline $\begin{array}{c}\text { Alta Eficiencia } \\
\text { Alta edad de lodo } \\
{\left[\mathrm{q}_{\mathrm{C}}>\mathbf{1 0} \text { días] }\right.} \\
\square\end{array}$ & $\begin{array}{c}\text { Mayor } \\
\text { consumo } \\
\text { de Oxígeno }\end{array}$ \\
$\begin{array}{c}\text { Menos lodo de exceso; lodo } \\
\text { más estable; }\end{array}$ & \\
$\begin{array}{c}\text { No es necesario: digestor de } \\
\text { lodos, sedimentador primario }\end{array}$ & Más energía \\
\hline
\end{tabular}

Tabla 2. Ventajas y desventajas de la aplicación de lodos activados por aireación extendida

Con un modelo biocinético más amplio es factible estudiar la necesidad de implementar un sedimentador primario en los lodos activados convencionales. Este requerimiento se motiva en el hecho de que ante una baja edad de lodo, la materia orgánica biodegradable particulada no logra transformarse por los microorganismos en el tanque de aireación, lo cual puede esperarse con la aireación extendida en vista de la alta edad del lodo y el considerable tiempo hidráulico de retención. 
El ejemplo aquí analizado posee una amplia pertinencia en clase, ya que brinda la oportunidad de explorar escenarios adicionales, entre ellos, la influencia de los parámetros del modelo sobre la eficiencia del proceso. Adicionalmente, puede servir para explicar y demostrar un procedimiento fundamental en los procesos biológicos, como es el mejoramiento de la eficiencia mediante el uso de un sedimentador secundario que permita separar la edad del lodo del tiempo hidráulico de retención. Todas estas aplicaciones son realizables en un tiempo mínimo con una herramienta computacional amigable.

\section{CONCLUSIONES}

Se describen los beneficios del uso de una herramienta computacional amigable para la modelación y simulación en el aprendizaje y práctica de la Ingeniería Ambiental. En un curso de Procesos Ambientales del currículo de la Ingeniería Ambiental se unen una gran cantidad de conceptos teóricos aprendidos previamente para la formulación de modelos de sistemas ambientales. Estos modelos, cuando involucran pocas variables de estado son sencillos y pueden solucionarse para condiciones dinámicas o de estado estacionario en forma analítica. Sin embargo, cuando son más complejos el uso de la solución numérica en una herramienta computacional como las mencionadas anteriormente es una gran ventaja en términos de tiempo y eficiencia en el aprendizaje. Adicionalmente, su uso puede acompañar el cubrimiento de la totalidad de un curso introductorio de modelación de sistemas ambientales que va desde los conceptos básicos de modelos matemáticos hasta la aplicación de conceptos como el análisis de incertidumbre.

La incorporación de programas computacionales amigables con el fin de implementar modelos matemáticos, representa una herramienta indispensable y de gran potencial en un curso de Procesos Ambientales. Su uso en el aula optimiza el tiempo destinado a reforzar los conceptos teóricos aprendidos por los estudiantes y propicia que éstos se concentren en las materias abarcadas y no en los detalles técnicos del programa empleado.

La herramienta seleccionada debe ser de fácil comprensión y sus resultados han de ser ofrecidos en una forma gráfica, cuya interpretación no genere dificultades. La principal fortaleza de este recurso es la utilización, en la definición de escenarios, de la pregunta ¿qué pasa si...?. De este modo, el estudiante observa lo que sucede al cambiar los parámetros del modelo. Su aplicación se ha evidenciado mediante un ejemplo de modelación de procesos biológicos para el tratamiento de aguas residuales

Debe prestarse especial atención a la planificación del curso. Con esta planeación es posible limitar las desviaciones en los objetivos y evitar que los estudiantes concentren su atención a la interpretación de los resultados más que a la implementación del código computacional. 


\section{REFERENCIAS BIBLIOGRÁFICAS}

Argent, R. M.(2004).An overview of model integration for environmental applications. Components, frameworks and semantics. Environmental Modeling and Software, vol. 19, No. 3 (March), pp: 219-234.

Bijlsma, M. and Hooijsman, T.(1996). Environmental process technology: IHE lecture notes EE 180/96/1. Delft, Holanda: IHE Delft. 100 p.

Brenner, A.; Shacham, M. and Cutlip, M, B.(2005). Applications of mathematical software packages for modeling and simulations in environmental engineering education. Environmental Modeling and Software, vol.20, No. 10 (October), pp.1307-1313.

Deaton, M.; and Winebrake, J. Dynamic modeling of Environmental Systems. Berlin: Springer Verlag, 2000. $191 \mathrm{p}$.

Egemen, E.; Edwards, F. and Nirmalakhandan, N.(1998). Computer simulation models in environmental education. Water Science and Technology, vol. 38, No.11, pp. 295-302.

Felder, R. M. (1988). Learning and teaching in engineering education. Engineering Education, vol.78, No.7, pp: 674-681.

Ford, A. Modeling the environment. Washington: Island Press, 2009. 375 p.

Gil-Rodríguez, M. Procesos de descontaminación de aguas- Cálculos informáticos avanzados. Sydney, Australia: Thomson, 2005. 261 p.

Gottfried, B. S. Spreadsheet Tools for Engineers Using Excel 2007. 3thed. New York: McGraw-Hill Science, 2009. $512 \mathrm{p}$.

Grady, L.; Daigger, G.; Love, N. and Filipe, C. (2011). Biological wastewater treatment. 3th Ed. Boca Raton, USA: CRC Press, 2011. 963 p.

Grau, P.; Sutton, P. M.;Henze, M.; Elmaleh, S.; Grady,C. P.; Gujer,W. and Koller, J.(1982). Recommended notation for use in the description of biological wastewater process. Water Research, vol.16, No. 11 (November), pp:1501-1505.

Gujer, W. and Henze, M. Activated sludge modeling and simulation. (1991). Water Science and Technology, vol. 23, No 4-6, pp: 1011-1023

Gujer, W. and Larsen, T. A. (1995). The implementation of biokinetics and conservation principles in ASIM. Water Science and Technology, vol. 31, No. 2, pp: 257-266. 
Gujer, W. (2000). Environmental engineering education at the Swiss Federal Institute of Technology in Zurich. Water Science and Technology, vol. 41, No. 2,pp: 37-45.

Gujer, W. (2004). Systems analysis in environmental engineering: how far should we go? Water Science and Technology, vol. 49, No.8, pp: 37-42.

Gujer, W. (2008). Systems analysis for water technology. Berlín: Springer, 2008. 459 p.

Henze, M.; Gujer, W.; Mino, T. and van Loosdrecht, M. C. M. Activated Sludge Models ASM1, ASM2, ASM2d, and ASM3. IWA Scientific and Technical Report no. 9. London: IWA Publishing, 2000.121 p.

Hug, T.; Benedetti, L.; Hall, E.; Johnson, B.; Morgenroth, E.; Nopens, I.; Rieger, L.; Shaw, A. and Vanrolleghem, P. (2009). Wastewater treatment models in teaching and training: The mismatch between education and requirements for jobs. Water Science and Technology, vol. 59, No. 4, pp:745- 753.

Ingham, J.; Dunn, I. J.; Heinzle, E.; Prenosil,J. E. and Snapee, J. B.(2008).Chemical Engineering Dynamics: An Introduction to Modelling and Computer Simulation. 3th Ed.New York: Wiley-VCH, 2008. 640 p.

Lawrence, A.; and McCarty, P. (1970). Unified basis for biological treatment design and operation. Journal of the Sanitary Engineering Division, 96(SA3P), pp: 757-778.

Macey, R. I.; Oster, G. F. and Zahney, T. (2001). Berkeley Madonna. Berkeley: University of California.

Makinia, J. (2010). Mathematical modeling and computer simulation of active sludge systems. Londres: IWA Publishing. 2010, 375 p.

Martínez, S. A. y Rodríguez, M. Tratamiento de aguas residuales con Matlab. Barcelona: Reverte. 2005, $237 \mathrm{p}$.

Metcalf, and Eddy. Wastewater engineering-treatment disposal reuse.4th Ed. New York: McGraw-Hill. 2003. 1819 p.

Nirmalakhandan, N.; Egemen, E. and Edwards, F. (1998). Comparison of software packages for developing simulation models. Water Science and Technology, vol. 38, No. 11, 315-322.

Nirmalakhandan, N. (2002). Modelling tools for environmental engineers and scientists. Boca Raton: CRC Press. 2002. 309 p.

Petersen, E.E. Chemical reaction analysis. New York: Prentice Hall - Englewood Cliffs. 1965. 276 p.

Reichert, P.(1995). Design techniques of a computer program for the identification of processes and the simulation of water quality in aquatic systems. Environmental Software, vol. 10, No. 3, pp: 199-210. 
Shacham, M. and Cutlip, M. B. (1999).A comparison of six numerical software packages for educational use in the chemical engineering curriculum. Computers in Education Journal, vol. 9, No. 3, pp: 9-15.

Shanahan, P.; Borchardt, D.; Henze, M.; Rauch, W.; Reichert, P.; Somlyódy, L and Vanrolleghem, P. (2001). River water quality model no. 1(RWQM1): I. Modeling Approach. Water Science and Technology, vol. 43, No. 5, pp: 1-9.

Schnoor, J, L. Environmental modeling- fate and transport of pollutants in water, air, and soil. New York: Wiley. 1996. 676 p.

TU-Delft, IHE-Delft.(2002). Short course on modeling of activated sludge wastewater treatment. Proceedings of the course.

Valsaraj, K. T. Elements of environmental engineering, thermodynamics and kinetics. 2th Ed. Boca Raton: Lewis.2000. 673 p.

Weber, W., DiGiano, F. Process dynamics in environmental systems. New York: Wiley Interscience. 1996. 925 p.

Weber, W. Environmental systems and processes. New York: Wiley Interscience.2000. 556 p. 